\title{
Lista preliminar da avifauna da A.P.A. Coqueiral e primeiro registro de Tytira inquisitor no sul de Minas Gerais, Brasil
}

\author{
Aloysio Souza de Moura ${ }^{1}$, Bruno Senna Correa ${ }^{2}$, Talita Vieira Braga ${ }^{3} \&$ Renato Gregorin ${ }^{4}$ \\ 1 Ornitólogo e graduando em Ciências Biológicas pelo Centro Universitário de Lavras "UNILAVRAS" \\ Caixa Postal 197, CEP 37.200.000, Lavras, MG. thraupidaelo@yahoo.com.br \\ 2 CEFET,MG-Campus IX-Nepomuceno Av. Monsenhor Luiz de Gonzaga, 103, Centro 37250-000 - Nepomuceno, \\ MG. bruno.senna@gmail.com \\ 3 Bióloga, graduada pela UFLA talitavbr@gmail.com \\ 4 Coordenador do Plano de manejo de fauna da APA Coqueiral, DBIO, setor de Zoologia, UFLA.” Lavras MG. \\ rgregorin@ufla.br
}

\section{RESUMO}

A diversidade de fauna é uma exigência para a criação de unidades de conservação. $O$ grupo de aves destaca-se por representar in loco parte dos parâmetros ecológicos que visam o estabelecimento de estratégias para a biologia da conservação. O presente trabalho teve por objetivo realizar um checklist preliminar de uma área, visando o estabelecimento de uma unidade de conservação (UC) do tipo APA (área de preservação ambiental), em ambiente fragmentado, margeado por cultivo agrícola, manchas de florestas e estradas estaduais. Foram registradas 205 espécies de aves alocadas em 52 famílias. Observou-se predomínio de representantes da família Tyrannidae (18,5\%) (N=38), seguido por Thraupidae (6 \%) $(\mathrm{N}=13)$, Furnaridae e Emberizidae (5,8 \% ambos) $(\mathrm{N}=12)$. Não foram registradas espécies com grau de ameaça de extinção. Os fragmentos florestais presentes na área de estudo registraram 45 espécies $(21,9 \%)$ da diversidade amostrada, destacando que estratos bem definidos (dossel, e. médio, e. baixo) estão favorecendo o deslocamento da avifauna. Observa-se fundamental o planejamento e execução de projetos que visem estratégias para a preservação da fauna local, na paisagem fragmentada.

Termos para indexação: levantamento de fauna, unidade de conservação, estratégias para preservação de espécies

\section{Preliminary bird species list of APA Coqueiral and first record of Tytira inquisitor in souther Minas Gerais State, Brazil}

\begin{abstract}
The fauna diversity is one of the requirements for the creation of units of conservation. The group of birds is distinguished for representing in leases part of the ecological parameters that aim at the establishment of strategies for the biology of the conservation. The present work had for objective to carry through one checklist preliminary of an area, aiming at the establishment of a unit of conservation (UC) of type APA (área de preservação ambiental), in antropic environment, bordered with agricultural culture, forest fragments and state roads. It had been registered 205 species of birds placed in 52 families. Predominance of representatives of the Tyrannidae family was observed $(18,5 \%)(\mathrm{N}=38)$, followed for Thraupidae $(6 \%)(\mathrm{N}=13)$, Furnaridae and Emberizidae $(5,8 \%$ both $)(\mathrm{N}=12)$. They had not been registered species with degree
\end{abstract}


of extinguishing threat. The forest fragments in the study area had registered 45 species $(21.9 \%)$ of the showed diversity, detaching that stratus well defined (canopy, medium strata, low strata) are favoring the displacement of avifauna. It observes basic the planning and execution of projects that aim at strategies for the preservation of the local fauna, in the fragmented landscape.

Indexed expressions: fauna survey, conservation unity, species preservation strategies

\section{INTRODUÇÃO}

Áreas naturais protegidas estabelecidas em terrenos particulares constituem um importante instrumento para a conservação da diversidade de aves, sendo um grande auxilio aos esforços públicos para a proteção da natureza.

O incentivo à criação de Áreas de Preservação Ambientais (A.P.A.) em comunidades rurais é considerada estratégica para a implantação de corredores ecológicos entre os fragmentos florestais remanescentes do Bioma Mata Atlântica e o Bioma Cerrado no sul do estado de Minas Gerais.

Desde a criação da primeira área protegida em 1872, milhares de quilômetros quadrados têm sido destinados a conservação, quase sempre por decisão dos órgãos ambientais. No entanto, o crescimento populacional, agricultura, minerações e plantio de Eucalipto, além da redução do orçamento público destinado à conservação da natureza, tem limitado cada vez mais a criação de áreas protegidas públicas (McNeely, 1984).

Ressalta-se a importância da compreensão sobre a avifauna, pois, o conhecimen- to sobre a composição das comunidades de grupos de vertebrados de diferentes áreas e a sua comparação são fatores relevantes em projetos de Biologia da Conservação (Lawton 1996).

O objetivo deste estudo é apresentar uma listagem preliminar da comunidade de aves da A.P.A. Coqueiral, fornecendo dados sobre a distribuição da avifauna no local e assim criar bases para futuros trabalhos de conservação.

\section{MATERIAL E MÉTODOS}

\section{Área de estudo}

A A.P.A. Coqueiral esta localizada no município de Coqueiral no sul do estado de Minas Gerais (23k 0463730/Utm 7666233). A paisagem é composta basicamente por áreas de cultivo (café, milho, feijão) e pastagens, pequenos fragmentos de Florestas Estacionais Semideciduais, Florestas Ciliares, Afloramento Rochoso (Figura 1), brejos (Figura 2) e uma pequena mancha de cerrado sensu strictu.

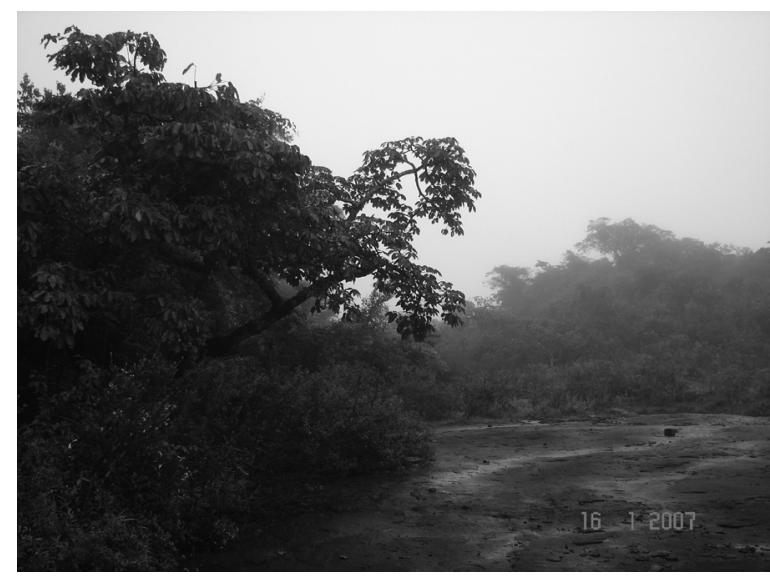

Figura 1. Afloramento rochoso (pedra do Ermo) (23k 0464244/Utm 7667790) 


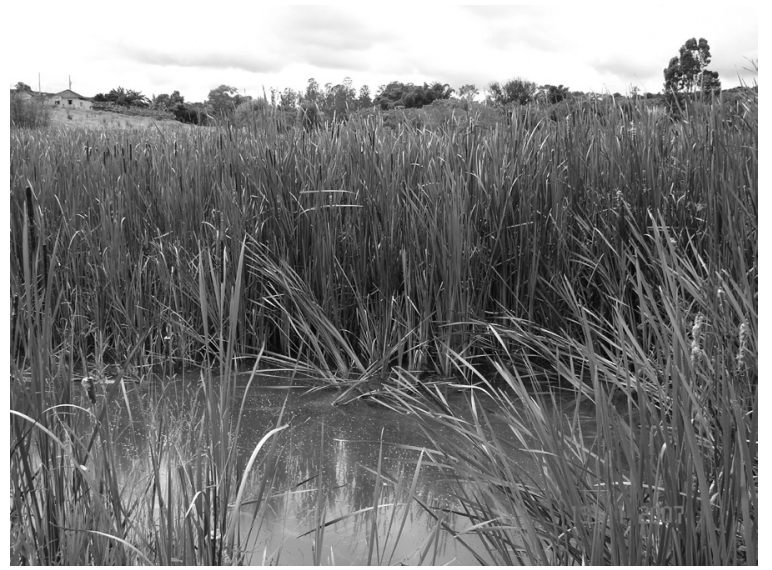

Figura 2. Brejo às margens do ribeirão Ermo (23k 0463341/Utm 7666127)

\section{Métodos}

Foi utilizado no presente trabalho a técnica da presença e ausência de espécies como único método de censo, pois o foco deste trabalho foi voltado a um inventário qualitativo.

As coletas de dados em campo abrangeram onze pontos da vegetação para melhor caracterização da área. Trilhas e estradas vicinais ou de serviço foram amostradas e percorridas a pé.

Os dados deste estudo foram coletados a partir de duas campanhas de campo junto à equipe do Laboratório de Zoologia da Universidade Federal de Lavras "UFLA" coordenada pelo Professor Renato Gregorin, uma primeira observação nas águas (Verão), realizada em 12/01/07 e teve seu término em 19/01/2007 e uma segunda campanha na seca (Inverno), realizada em 25/04/2007 e teve seu término em 03/05/2007.

As espécies foram observadas e identificadas com o auxilio de binóculos Onida 10x50 e de guias de identificação de campo: Belton (2004), Develey (2004), Major Jr. et al. (2004) e Souza (2004). Sempre que possível a sonorização das aves foi registrada com auxilio de um gravador Panasonic VAS modelo RN305 em fita K7 60 minutos, também para fins de identificação da espécie. As espécies coletadas em redes de neblina foram fotografadas com o auxilio de uma câmera da marca Sony modelo H1 e soltas no mesmo momento, sem que houvesse qualquer prejuízo para o indivíduo (figura 3,4 e 5). Os pontos de observação foram marcados em GPS da marca Garmin modelo ETREX.

Nem todas as aves foram identificadas no local. Alguns exemplares tiveram sua vocalização gravada em áudio, quando possível, e sua fotografia registrada, a fim de uma identificação posterior, seja pela consulta em museus, seja pelo contato com ornitólogos.

A metodologia empregada para o levantamento da ornitofauna desta área constitui-se basicamente em princípios de amostragem. O primeiro, conclusivo, baseando no contato direto do pesquisador com o objeto da pesquisa, levando-o a identificação dos exemplares (Método Animal-focal). Já o segundo principio, unicamente indicativo, foi orientado na obtenção de indícios que posteriormente poderão levar o pesquisador a comprovação de sua ocorrência. O desenvolvimento destes princípios foi realizado através de técnicas diversas, visto a grande heterogeneidade do grupo em estudo.

Durante as observações da avifauna em cada ponto de amostragem da área o pesquisador esperava 30 minutos para amenizar o impacto do observador com o meio. Após este período, foram realizados os seguintes procedimentos:

1-Contato visual: esta técnica foi desenvolvida percorrendo a área compreendida pelo ponto de amostragem com a finalidade de registrar espécies ocorrentes na área e sua posterior identificação.

2-Contato auditivo: A presença de algumas espécies, na maioria das vezes, só puderam ser evidenciadas por esta técnica, quer pelo reconhecimento direto da vocalização de determinada espécie, como pela análise posterior conseguida pela gravação de voz, em fita K7. O emprego 
do gravador auxiliou no contato de espécies arredias, que são atraídas pela própria vocalização ("play-back").

3-Vestígios: Dentro desta categoria incluíram-se quaisquer indícios que denotassem a ocorrência de aves em determinado local, a saber, tais como penas, ninhos, restos alimentares, fezes, pegadas, entre outros.

4-Relatos: Este método foi aplicado através de diálogos e entrevistas com indivíduos da população local ligados ao meio silvestre como: lenhadores, pescadores e moradores da região. No caso do presente trabalho os relatos serviram para confirmar e direcionar os trabalhos de campo, não sendo utilizados como registro para espécies não observadas ao longo das observações.

5-Captura com redes de neblina: este procedimento teve como base auxiliar na identificação das espécies de aves arredias que vivem entre a ramaria da floresta (estrato baixo, solo) e quase nunca sonorizam.

Todas as fases de coleta com redes de neblina foram conduzidas com a duração de um dia completo (amanhecer ao entardecer) e de algumas noites $(16: 30 \mathrm{~h}-22: 00 \mathrm{~h})$ junto a equipe do laboratório de Zoologia da Universidade Federal de Lavras que inventariava Quirópteros naquela área. As observações com binóculos tiveram horários variados mais de modo que todos os pontos de amostragem tiveram coleta de dados em três horários de maior importância do dia (amanhecer, meio do dia, entardecer). Foram contabilizados 17 dias de trabalho de campo, que somaram 200 horas totais de coleta de dados em campo.

A nomenclatura das espécies seguiu o Comitê Brasileiro de Registro Ornitológicos (CBRO, 2007) e informações sobre comportamento migratório e alimentar seguiu Sick (2001) e Sigrist (2007 \& 2009).

Para as análises de riqueza estimada de espécies, foi utilizado um método não-paramétrico do tipo Jacknife 1 (de primeira ordem) (Krebs, 1989; Palmer, 1990). A análise foi feita com o auxílio do programa PAST.

$$
\text { Jack 1 }=\mathrm{S}+\mathbf{L} \cdot \frac{\mathbf{m}-\mathbf{1}}{\mathbf{m}}
$$

$\mathrm{S}=$ número de espécies observadas

$\mathrm{L}=$ número de espécies registradas em uma amostra

$\mathrm{m}=$ número de amostras

$\mathrm{m}-1=$ número de amostras -1

Foi calculado o índice de diversidade de espécies pela estimativa de Shannon-Wiener ( $\left.\mathrm{H}^{\prime}\right)$, segundo Magurran (1988), para a comunidade.

$$
\mathbf{H}^{\prime}=-\Sigma \text { Piln (Pi) }
$$

$\mathrm{Pi}=$ proporção dos indivíduos da espécie i em relação ao número total de indivíduos da comunidade

Foram realizadas Análises de Agrupamento Hierárquico (cluster) de similaridade entre ambientes, a partir de dados de abundância de espécies com índice de similaridade de Bray Curtis. Os agrupamentos mais evidentes foram destacados e comparados com os resultados do DCA (Detrended Correspondence Analysis).

\section{RESULTADOS E DISCUSSÃO}

Foram registradas 205 espécies de aves para a A.P.A. Coqueiral, alocadas em 52 famílias (tabela 1 em anexo). Em relação à distribuição, observou-se predomínio de representantes da família Tyrannidae $(18,5$ $\%)(\mathrm{N}=38)$, seguido por Thraupidae (6\%) $(\mathrm{N}=13)$, Furnaridae e Emberizidae $(5,8 \%$ ambos) $(\mathrm{N}=12)$.

Já era esperado que a família Tyrannidae fosse a mais abundante em espécies, por ser esta a família com maior numero de representantes na América do sul (Sick, 2001), corroborando outros trabalhos de inventario ornitológicos no sul de Minas 
Gerais (D'Angelo Neto et al. 1996, 1998, Vasconcelos 2002, Lombardi et al. 2007, Corrêa 2008). As espécies mais frequentemente capturadas no presente trabalho foram: Conopophaga lineata, P. mystaceus, Chiroxiphia caudata (Figura 3), Sittasomus griseicapillus (Figura 4) Basileuterus hypoleucus e B. flaveolus (Figura 5).

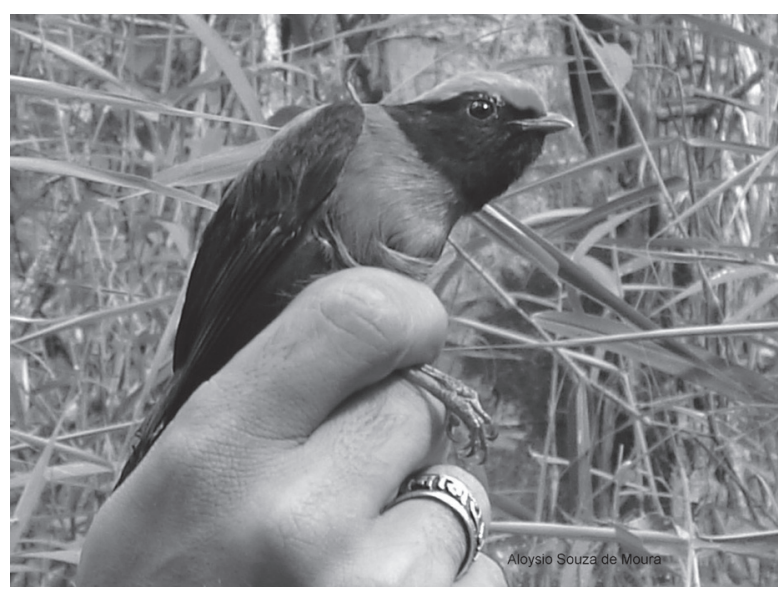

Figura 3. Chiroxiphia caudata Foto: Aloysio Souza de Moura

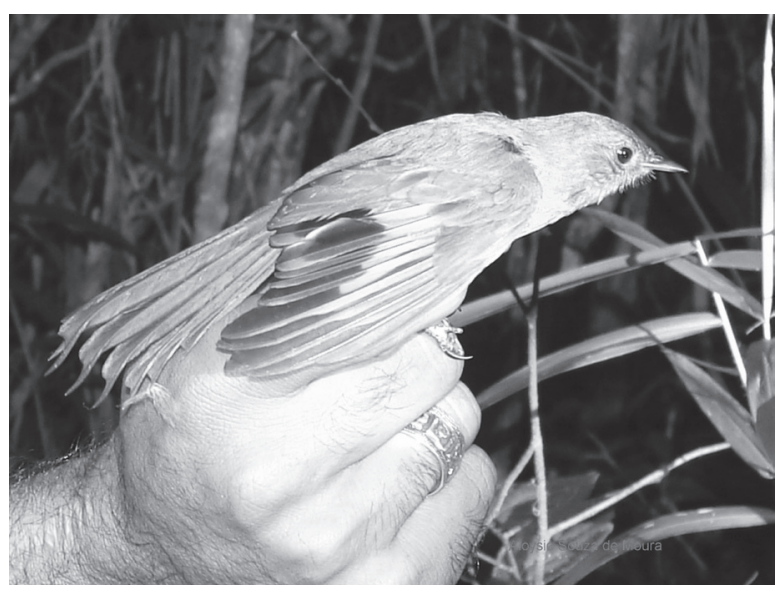

Figura 4. Sittasomus griseicapillus Foto: Aloysio Souza de Moura

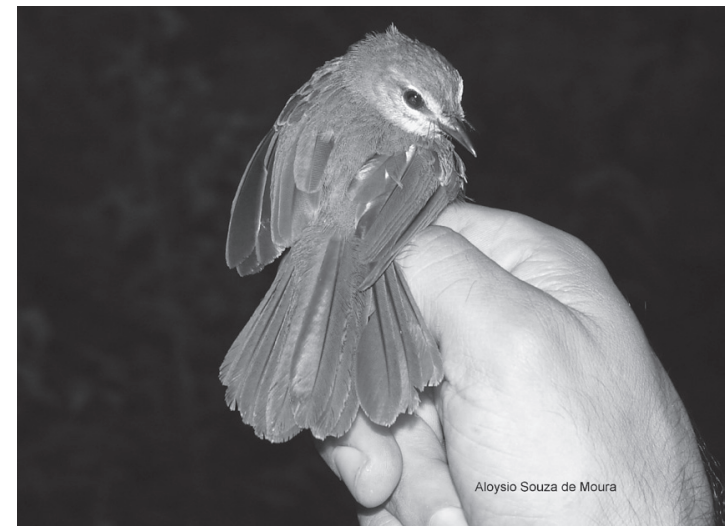

Figura 5. Basileuterus flaveolus Foto: Aloysio Souza de Moura

A fitofisionomia com maior abundância de espécies foi as áreas abertas com 49,7\% das espécies $(\mathrm{N}=102)$, acompanhado dos fragmentos de Florestas Semideciduais com 46,3\% $(\mathrm{N}=95)$, Matas ciliares 44,3\% ( $\mathrm{N}=91)$ e Afloramento Rochoso 40,4\% ( $\mathrm{N}=83)$. A fitofisionomia de cerrado sensu strictu apresentou 21,4\% de registro de espécies $(\mathrm{N}=44)$ (Gráfico 1).

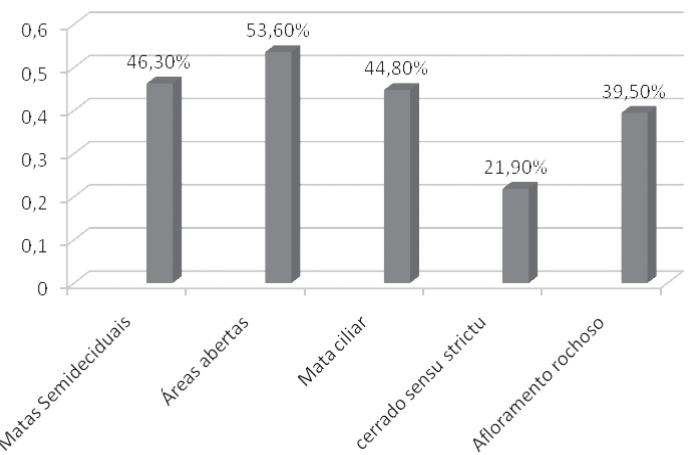

Gráfico 1. Distribuição das espécies de aves nas áreas da A.P.A. Coqueiral, MG

O motivo de ter se registrado o maior numero de aves em áreas abertas deve-se o fato de que esta e a maior paisagem das áreas da A.P.A. Coqueiral, e o baixo número de registro para o cerrado sensu strictu pode estar relacionado à área reduzida da área de estudo (menor que 20 ha). 
Das 205 espécies de aves registradas na área inventariada 7,8\% das espécies $(\mathrm{N}=16)$ são abundantes, pois, foram registradas em todas as fitofissionomias amostradas (tabela 1), são aves "generalista de habitats" e comuns na A.P.A.

Este é o primeiro inventário de aves da A.P.A. Coqueiral, mas, estando ciente de que nenhuma área por mais bem amostrada que esteja, terá sua fauna completamente conhecida, pois, listas regionais não são estáticas e sim muito dinâmicas por motivos climáticos ou por atividades humanas (Vanconcelos et al., 2003) espera-se trabalhos futuros para atualização constante do conhecimento da composição da avifauna da A.P.A., pois, no caso de aves estas variações em listas de espécies tem sido registrada em varias localidades do país (Willis \& Oniki, 1993; Sick, 2001; Machado et al., 1998).

Embora não tenha sido registrada nenhuma espécie de aves que sofra ameaça de extinção a A.P.A. Coqueiral mostra-se importante para conservação do grupo estudado, pois, em relação a localização da área e as condições atuais da vegetação natural o numero de espécies se mostrou bastante alto em relação as aves descritas para o território Brasileiro, 11,3\% das 1801 espécies descritas para o país(CBRO, 2007).

Os fragmentos de Mata Semidecidual denominados Mata do Pedrinho (23k 0464377/Utm 7664097) e Mata do Carlos (23k 0464240/Utm7666810) se mostraram importante para a comunidade local de aves, pois, $46,3 \%$ das espécies foram registradas nestes pontos e 45 espécies $(21,9 \%)$ somente nestes pontos (gráfico 2), o alto numero de espécies se deve pelo motivo da grande variedade vegetal com três estratos bem definidos (dossel, e. médio, e. baixo) e também se ter usado e técnica de contato auditivo associado a gravações (caso de duvida) para identificação das espécies, indicando a importância do conhecimento prévio das vozes de cada espé- cie para realização de inventários em áreas de florestas tropicais (Sick, 2001).

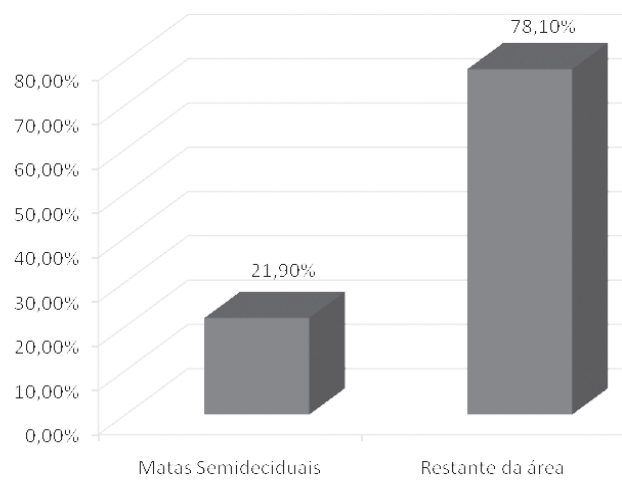

Gráfico 2. Aves somente registradas nas Matas do Pedrinho e na Mata do Carlos em relação ao restante dos pontos

A estimativa de riqueza pelo método de Jacnkife $1=287,8$. Resultados em ambientes similares, no sul de Minas Gerais podem ser observados na tabela 2 .

Tabela 2. Estimativa de riqueza de aves no Sul de Minas Gerais

\begin{tabular}{|l|l|}
\hline Referência & Jack1 \\
\hline Corrêa (2008) & 146,25 \\
\hline Corrêa \& Moura (2009) & 232,65 \\
\hline Corrêa \& Moura (2009) & 179,67 \\
\hline
\end{tabular}

Os índices de diversidade de espécies (Shannon Wiener)(H') foi $\mathrm{H}^{\prime}=4,554$ (matas semidecíduas)(MS), $\mathrm{H}^{\prime}=4,7$ (áreas abertas)(AA), $\mathrm{H}^{\prime}=4,5$ (mata ciliar)(MC), $\mathrm{H}^{\prime}=3,8$ (cerrado)(C), $\mathrm{H}^{\prime}=4,3$ (afloramento rochoso) (AR). Estimativas realizadas nas mesmas condições, em ambientes similares no sul de Minas Gerais podem ser observados na tabela 3 .

Tabela 3. Índice de diversidade de aves (H') no Sul de Minas Gerais

\begin{tabular}{|l|l|l|l|l|l|}
\hline Referência & MS & AA & MC & C & AR \\
\hline Corrêa (2008) & 3,23 & 2,5 & 3,13 & 3,5 & - \\
\hline $\begin{array}{l}\text { Corrêa \& } \\
\text { Moura (2009) }\end{array}$ & 4,4 & 3,8 & 4,5 & 4 & - \\
\hline $\begin{array}{l}\text { Corrêa \& } \\
\text { Moura (2009) }\end{array}$ & - & 4,65 & 4,47 & 4,5 & 4,89 \\
\hline
\end{tabular}


A permeabilidade da área aberta pode ser um dos fatores que influenciam alguns desses índices. Alguns valores mais elevados, para ambientes fragmentados e reduzidos podem estar relacionados com a presença de espécies generalistas.

O dendrograma de similaridade (cluster) (gráfico 3) formou 2 grandes grupos: 1. (AA, AR e C) (60\%); 2. (MC e MS)(48\%). $\mathrm{O}$ primeiro grupo apresentou maior similaridade entre AA e AR (69\%).

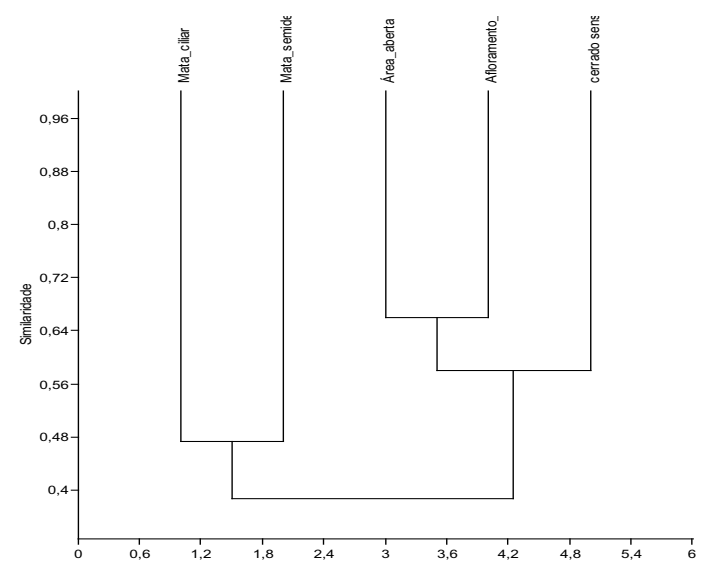

Gráfico 3. Cluster da composição da avifauna nos ambientes amostrados

A análise de DCA auxiliou no posicionamento dos elementos da paisagem (5 grupos) (gráfico 4) (tabela 3).

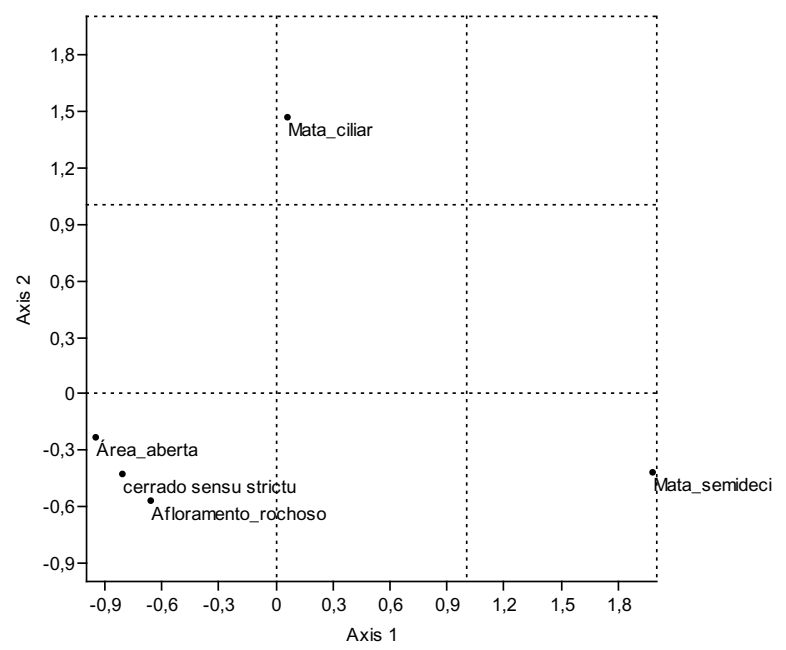

Gráfico 4. Distribuição da avifauna nos ambientes amostrados - DCA
Tabela 3. Valores de Eigen da análise de DCA da freqüência relativa nos ambientes amostrados

\begin{tabular}{|l|r|r|r|}
\hline & \multicolumn{1}{|l|}{ Eixo 1 } & Eixo 2 & \multicolumn{1}{|l|}{ Eixo 3 } \\
\hline $\begin{array}{l}\text { Matas } \\
\text { Semideciduais }\end{array}$ & $\mathbf{1 , 9 7 9 7}$ & $-0,42275$ & $-0,1274$ \\
\hline Áreas abertas & $-0,94718$ & $-0,23736$ & $-0,75892$ \\
\hline Mata ciliar & 0,06276 & $\mathbf{1 , 4 5 5 8}$ & 0,1514 \\
\hline Cerrado & $-0,80873$ & $-0,43606$ & $\mathbf{0 , 3 2 8 7 6}$ \\
\hline $\begin{array}{l}\text { Afloramento_- } \\
\text { rochoso }\end{array}$ & $-0,65676$ & $-0,57507$ & $\mathbf{0 , 8 2 7 3 2}$ \\
\hline
\end{tabular}

A diversidade das espécies registradas nos ambientes de AA, C e AR está relacionada com a presença de espécies generalistas, com maior capacidade de dispersão entre os ambientes. A lista de espécies registradas relacionada ao impacto antrópico dos ambientes avaliados, entre outros, permitem caracterizar a diversidade da taxocenose de aves em escala local (Corrêa, 2008).

Em relação aos fragmentos florestais, a composição da avifauna parece estar relacionado com presença de espécies associados a formações em estado avançado de regeneração.

O Azulão (Cyanocompsa passerinus), a Juruva Verde (Barypthengus ruficapillus), Patativo chorão (Sporophila leucoptera) e o Anambé branco (Tytira inquisitor) tiveram apenas um registro em todas as campanhas de campo, sugerindo assim como espécies localmente raras pela sua baixa ocorrência na área durante as coletas de dados.

Das espécies registradas nas campanhas verão (Janeiro), 11 espécies estão associadas a habitats aquáticos, todas de vasta distribuição no país: Irerê (Dendrocygna viduata), Marrequinho (Dendrocygma autumnalis), Pé-vermelho (Amazoneta brasiliensis), Mergulhão (Tachybaptus dominicus), Socó-boi (Tigrisoma lineatum), Garça (Ardea alba), Frango-d'água(Galinula chloropus), Jaçanã (Jacana jacana), Martim-pescador (Megaceryle torquata), Martim-pescador (Chorocerile americana), Garibaldi (Chrysomus ruficapillus). 
Considerando o numero de espécies (tabela 1), nota-se que a estrutura trófica não varia muito de uma fitofissionomia a outra, com exceção dos Cafezais, onde faltam frugívoros, nectarívoros e insetívoros de troncos, provavelmente por não terem recursos disponíveis neste tipo de cultivo que é baseado em monodominância.

Apesar de uma grande porção da área da A.P.A. Coqueiral ser localizada em áreas de cultivo (agricultura), ambientes fragmentados têm se mostrado necessário para o estabelecimento, deslocamento, utilização e as vezes nidificação de várias espécies de aves (Blake et al, 1990; Blake \& Loissele, 1991). Petit \& Petit (2003) estudaram a importância de varias áreas cultivadas para a conservação de aves neotropicais, reconhecendo que plantações que conferem um grau de sombreamento ao ambiente (ex. Café) são importantes por abrigar espécies de relacionados a diferentes ambientes, entre elas florestais generalistas e especialistas de arbustos e áreas abertas.

Os resultados embora sejam preliminares, levantam a necessidade de projetos de conservação na área de estudo.

\section{Registros Interessantes}

Cyanocorax crysops, no dia 27 de abril de 2007 foi visualizado e registrado fotograficamente um casal de Gralha picaça na mata ciliar do ribeirão Ermo junto a varias Gralhas do Cerrado (Cyanocorax cristatellus), está espécie só foi registrada esta única vez na A.P.A. e seu co-genérico gralha do cerrado é muito comum na área e na região.

Tytira inquisitor. Apesar de sua vasta distribuição (Sick, 1997, Souza, 2004) apenas um registro foi feito desta espécie nas áreas da A.P.A., este é o primeiro registro documentado no sul do estado de Minas Gerais deste Anambé (Lombardi et al. 2007, Vasconcelos, 2002.; Ribon, 2000., D’AngeloNeto et al. 1996,1998). Um casal foi visualizado em uma árvore emergente na borda do fragmento de Mata do Carlos no dia 17 de janeiro de 2007.

Cyanocompsa passerinus. Mesmo sendo descrita como ocorrente no sul de Minas Gerais o Azulão vem tendo suas populações reduzidas na região devido ao uso de agrotóxicos e à captura por passarinheiros. No dia 15 de janeiro de 2007 foi visualizado um Azulão macho com plumagem jovem em um brejo as margens do ribeirão Ermo próximo a uma plantação de arroz.

Considerando a carência de informações sobre a fauna regional do Sul de Minas Gerais, enfatizamos que o conhecimento da composição regional da fauna é essencial para preencher lacunas na proteção da biodiversidade, criando novas áreas de proteção ambiental que incluam espécies mal representadas em outras áreas de conservação.

\section{Agradecimentos}

Ao professor Vicente Gualberto (Dep. De Solos) da UFLA, Sr. Luiz (Emater) de Coqueiral pelo apoio logístico das campanhas. A Prefeitura Municipal de Coqueiral pelas acomodações e apoio, e aos colegas de equipe Silvia Maiani, Shayene, Elisandra Chiquito, Arthur Tahara, Ricardo Augusto Ceborcini e Daniel Quedes pelos esforços no campo, dia e noite. Aos moradores da comunidade do Ermo que sempre permitiram a entrada da equipe em suas propriedades sem nenhum problema.

\section{REFERENCIAL BIBLIOGRÁFICO}

\author{
BELTON, W. Aves Silvestres do Rio Gran- \\ de do Sul. $4{ }^{\text {a }}$ ed. Porto Alegre. Ed. Fundação \\ Zoobotânica do Rio Grande do Sul, 2004. \\ $175 \mathrm{p}$.
}


BLAKE, J.G.; LOISSELE, B.A.; MOERMOND, TC.; LEVEY, D.J.; DENSLOW, J.S. Quantifying abundance of fruits for birds in tropical habitats. Studies in Avian Biology, Los Angeles, n.13, p. 73-79, 1990.

BLAKE, J.G.; LOISSELE, B.A. Variation in risourcer abundance affects capture rates of birds in three lowland habitats in Costa Rica. Auk, Lawrence, n. 108, p. 114-130, 1991.

COMITÊ BRASILEIRO DE REGISTROS ORNITOLÓGICOS. Listas das aves do Brasil. Versão 16.8/2007. Disponível em: $<$ http://www.cbro.org.br>. Acesso em: 20 jan. 2007.

CORRÊA, B.S. Avifauna em fragmentos florestais conectados por corredores ecológicos no município de Lavras - Minas Gerais. 2008. 132p. Tese (Doutorado em Engenharia Florestal) - Universidade Federal de Lavras.

CORRÊA, B.S.; MOURA, A.S. MATAVELLI, R. Levantamento de herpetofauna, avifauna e mastofauna na área de exploração, área de influência direta e área de influência indireta para composição de EIA-Rima da empresa de Mineração Cal Ferreira. 2009. 62 p.

CORRÊA, B.S.; MOURA, A.S.; FERREIRA e SILVA, G. Levantamento preliminar de herpetofauna, avifauna e mastofauna para composição de Meio Biótico para Planejamento de Unidade de Conservação no município de Perdões, MG. 2009. 64 p.

D’ÂNGELO NETO, S. Levantamento e caracterização da avifauna do campus da UFLA. 1996. 58 p. Dissertação (Mestrado em Engenharia Florestal) Universidade Federal de Lavras.
D’ÂNGELO NETO, S.; VENTURIM, N.; OLIVEIRA-FILHO, A.T.; COSTA, F.A.F. Avifauna de quatro fisionomias florestais de pequeno tamanho (5 a 8 ha) no campus da UFLA. Revista Brasileira de Biologia, São Carlos, v. 58, n. 3, p. 463-472. 1998.

DEVELEY,P.F. Aves da grande São Paulo. São Paulo. Ed. Aves e Fotos. 2004. 299p.

KREBS, C.J. Ecological methodology. [S.1.]: Harper Collins, 1989. 654 p.

LAWTON, J.H. Population abundance, geographic range and conservation: 1994 Witherby lecture. Bird Study n. 43, p.3-19,1996.

LOMBARDI, V.T., VASCONCELOS, M.F., D’ÂNGELO NETO, S.(2007). Novo registros ornitológicos para o Centro-Sul de Minas Gerais (alto Rio Grande): municípios de Lavras, São João Del Rei e adjacências, com a listagem revisada da região. Atualidades Ornitológicas on-line. $\mathrm{n}^{\circ} 139$ - www.ao.com.br

MACHADO, R.B.; RIGUEIRA, S.E.; LINS, L.V.(1998). Expansão geográfica do canário rabudo (Embernagra lougicalda- Aves, Emberizidae) em Minas Gerais. Ararajuba, n. 6, p. 42-45, 1998.

MAGURRAN, A. Ecological diversity and its measurement. Cambridge: University of Cambridge, 1988. 179 p.

MAJOR,I.; Jr SALES, L.G.; CASTRO, R. Aves da caatinga. Fortaleza. Ed. Demócrito Rocha. Associação caatinga, 2004. 256p.

McNEELY, J. A. Protected áreas are new realities. In McNEELY, J A; MILLER, K R eds. National parks,conservation, and development adapting to: the role of protected areas in sustaining society. USA, Smithsonian, p.1-7. 1984. 
PALMER, M.W. The estimations of species diversity. Annual Review of Ecology and Systematics, v. 5, p. 285-307, 1990.

PETIT, L.S.; PETIT ,D.R. Evaluating the importance of human-modified lands for neotropical bird conservation. Conservation Biology.Montpelier. v. 17, n. 3, p. 687-694. 2003.

RIBON, R. Lista Preliminar da avifauna do Municipio de Ijací, Minas Gerais. Revista Ceres. v. 47, n. 274, p.665-682, 2000.

SICK,H. Ornitologia Brasileira. Rio de Janeiro: Editora Nova Fronteira. 2001.

SIGRIST, T. Aves do Brasil Oriental. São Paulo: Editora Avis Brasilis. 2007.

SIGRIST, T. Avifauna Brasileira. São Pau1o: Editora Avis Brasilis. 2009.

SOUZA,D. Todas as Aves do Brasil. Feira de Santana: Ed.Dall. 350p. 2004.
VASCONCELOS, M.F.; D’ÂNGELO NETO S.; BRAND; VENTURIM, N.; OLIVEIRA-FILHO, A.T.; COSTA, F.A.F. Avifauna de Lavras e municípios adjacentes, Sul de Minas Gerais, e comentários sobre sua conservação. Unimontes Cientifica, Montes Claros, v.4, n.2, p. 153-165. 2002.

VASCONCELOS, M.F., VASCONCELOS, P.N., MAURÍCIO, G.N., MATRANGOLO, C.A.R., DELL'AMORE, C.M., NEMÉSIO, A., FERREIRA, J.C.; ENDRIGO, E. Novos registros ornitológicos para a Serra do Caraça, com comentários sobre distribuição geográfica de algumas espécies. Lundiana, Belo Horizonte, v.4, n. 2, p. 135-139. 2003.

WILLIS, E.O. \& ONIKI, Y. New and reconfirmed from the state of São Paulo, Brazil, whit notes on disappearing specie. Bulletin of the British Ornithologists' club, n. 113, p. 23-34. 1993. 
Tabela 01: Lista de espécies de aves ocorrentes em 2007 na A.P.A. Coqueiral MG

\begin{tabular}{|c|c|c|c|c|c|c|c|}
\hline Família & Nome popular & Nome científico & Matas Semideciduais & Áreas abertas & Mata ciliar & cerrado sensu stricto & Afloramento rochoso \\
\hline Tinamidae & Inhambu guaçú & Crypturellus obsoletus & $\mathrm{x}$ & & & & \\
\hline Tinamidae & chororó & Crypturellus parvirostris & & $\mathrm{x}$ & & $\mathrm{x}$ & \\
\hline Tinamidae & Perdiz & Rhynchotus rufescens & & $\mathrm{x}$ & & $\mathrm{x}$ & \\
\hline Anatidae & Irerê & Dendrocygma viduata & & $\mathrm{x}$ & $\mathrm{x}$ & & \\
\hline Anatidae & Marrequinho & Dendrocygma autumnalis & & & $\mathrm{x}$ & & \\
\hline Anatidae & Pé vermelho & Amazonetta brasiliensis & & & $\mathrm{x}$ & & \\
\hline Cracidae & Jacu pemba & Penélope superciliaris & $\mathrm{x}$ & $\mathrm{x}$ & $\mathrm{x}$ & & $\mathrm{x}$ \\
\hline Cracidae & Jacuaçu & Penélope obscura & $\mathrm{x}$ & & & & $\mathrm{x}$ \\
\hline Podicipedidae & Mergulhão & Tachybaptus dominicus & & & $\mathrm{x}$ & & \\
\hline Ardeidae & Socó boi & Tigrisoma lineatum & & & $\mathrm{x}$ & & \\
\hline Ardeidae & Garcinha & Bubulcus ibis & & $\mathrm{x}$ & & & \\
\hline Ardeidae & Garça & Ardea alba & & $\mathrm{x}$ & $\mathrm{x}$ & & \\
\hline Ardeidae & $\mathrm{M}^{\mathrm{a}}$ Faceira & Syrigma sibilatrix & & $\mathrm{x}$ & & & \\
\hline Threskiornithidae & Curicaca & Theristicus caudatus & & $\mathrm{x}$ & & & \\
\hline Cathartidae & Urubu campeiro & Cathartes aura & & $\mathrm{x}$ & & $\mathrm{x}$ & $\mathrm{x}$ \\
\hline Cathartidae & Urubu & Coragyps atratus & $\mathrm{x}$ & $\mathrm{x}$ & & $\mathrm{x}$ & $\mathrm{x}$ \\
\hline Cathartidae & Urubu rei & Sarcoramphus papa & & & & & $\mathrm{x}$ \\
\hline Accipitridae & Gavião peneira & Elanus leucurus & & $\mathrm{x}$ & & & $\mathrm{x}$ \\
\hline Accipitridae & Gavião terra & Heterospizias meridionalis & & $\mathrm{x}$ & & $\mathrm{x}$ & $\mathrm{x}$ \\
\hline Accipitridae & Gavião carijó & Rupornis magnirostris & $\mathrm{x}$ & $\mathrm{x}$ & $\mathrm{x}$ & $\mathrm{x}$ & $\mathrm{x}$ \\
\hline Accipitridae & Gavião & Buteo albicaldatus & & $\mathrm{x}$ & & & $\mathrm{x}$ \\
\hline Acicipitridae & Gavião & Buteo albonotatus & $\mathrm{x}$ & & & & \\
\hline Falconidae & Falcão caburé & Micrastrur ruficollis & $\mathrm{x}$ & & & & \\
\hline Falconidae & Quiriquiri & Falco sparverius & & $\mathrm{x}$ & & $\mathrm{x}$ & $\mathrm{x}$ \\
\hline Falconidae & Falcão coleira & Falco femoralis & $\mathrm{x}$ & & & & \\
\hline Falconidae & Carrapateiro & Milvago chimachima & & $\mathrm{x}$ & & $\mathrm{x}$ & $\mathrm{x}$ \\
\hline Falconidae & Caracara & Caracará plancus & & $\mathrm{x}$ & & $\mathrm{x}$ & $\mathrm{x}$ \\
\hline Falconidae & Acauã & Herpetotheres cachinnans & & $\mathrm{x}$ & & & \\
\hline Rallidae & Saracura & Aramides cajanea & & & $\mathrm{x}$ & & \\
\hline Rallidae & Frango d'agua & Galinula chloropus & & & $\mathrm{x}$ & & \\
\hline Cariamidae & Seriema & Cariama cristata & & $\mathrm{x}$ & & $\mathrm{x}$ & \\
\hline Charadridae & Quero quero & Vanellus chilensis & & $\mathrm{x}$ & & & \\
\hline Jacanidae & Jaçanã & Jaçanã jacana & & & $\mathrm{x}$ & & \\
\hline Columbidae & Rolinha & Columbina talpacoti & & $\mathrm{x}$ & $\mathrm{x}$ & $\mathrm{x}$ & $\mathrm{x}$ \\
\hline Columbidae & Fogo p'ago & Columbina squamata & & $\mathrm{x}$ & & & \\
\hline Columbidae & Trocal & Patagioenas picazuro & & $\mathrm{x}$ & $\mathrm{x}$ & & \\
\hline Columbidae & Pomba de bando & Zenaida auriculata & & $\mathrm{x}$ & & & \\
\hline Columbidae & Juriti jemedeira & Leptotila rufaxila & $\mathrm{x}$ & & & & \\
\hline Columbidae & Juriti pupu & Leptotila verreauxi & $\mathrm{x}$ & & $\mathrm{x}$ & & \\
\hline Psttacidae & Perequitão & Aratinga leucophitalma & & $\mathrm{x}$ & $\mathrm{x}$ & $\mathrm{x}$ & $\mathrm{x}$ \\
\hline Psttacidae & Jandaia & Aratinga auricapillus & & $\mathrm{x}$ & & & $\mathrm{x}$ \\
\hline Psttacidae & Perequito rei & Aratinga aurea & & $\mathrm{x}$ & & $\mathrm{x}$ & $\mathrm{x}$ \\
\hline Psttacidae & Perequito & Forpus xanthopterygius & & $\mathrm{x}$ & & & $\mathrm{x}$ \\
\hline Psttacidae & Perequito & Brotogeris chiriri & & $\mathrm{x}$ & $\mathrm{x}$ & & \\
\hline Psttacidae & Papagaio & Pionus maximiliani & & & & & $\mathrm{x}$ \\
\hline Cuculidae & Alma de gato & Piaya cayana & $\mathrm{X}$ & $\mathrm{x}$ & $\mathrm{x}$ & $\mathrm{x}$ & $\mathrm{x}$ \\
\hline Cuculidae & Anu preto & Crotophaga ani & & $\mathrm{x}$ & $\mathrm{x}$ & & \\
\hline
\end{tabular}

Continua..... 
Lista preliminar da avifauna da A.P.A. Coqueiral e primeiro

registro de Tytira inquisitor no Sul de Minas Gerais, Brasil

Tabela 1, cont.

\begin{tabular}{|c|c|c|c|c|c|c|c|}
\hline Família & Nome popular & Nome científico & Matas Semideciduais & Áreas abertas & Mata ciliar & cerrado sensu stricto & Afloramento rochoso \\
\hline Cuculidae & Anu branco & Guira guira & & $\mathrm{X}$ & & & \\
\hline Cuculidae & Saci & Tapera naevia & $\mathrm{X}$ & & $\mathrm{X}$ & & \\
\hline Tytonidae & Coruja de igreja & Tyto alba & & $\mathrm{x}$ & & & \\
\hline Strigidae & Corujinha & Megascops choliba & $\mathrm{X}$ & & & & \\
\hline Strigidae & Murucutu & Bubo virginianus & & & & & $\mathrm{X}$ \\
\hline Strigidae & Coruja orelhuda & Rhinoptynx clamator & $\mathrm{X}$ & & & & \\
\hline Strigidae & Buraqueira & Athene cunicularia & & $\mathrm{x}$ & & & \\
\hline Nyctibiidae & Urutal & Nyctibius griseus & $\mathrm{X}$ & & & & \\
\hline Caprimulgidae & Bacurau & Nyctidromus albicollis & $\mathrm{X}$ & $\mathrm{X}$ & & & $\mathrm{X}$ \\
\hline Caprimulgidae & Bacurau & Caprimulgus parvulus & & & & & $\mathrm{X}$ \\
\hline Apodidae & Andorinhão & Streptoprocne zonaris & & $\mathrm{X}$ & & & $\mathrm{X}$ \\
\hline Apodidae & Andorinha temporal & Chaetura meridionalis & & $\mathrm{X}$ & & & $\mathrm{X}$ \\
\hline Trochilidae & Rabo branco peq. & Phaetornis ruber & $\mathrm{X}$ & & & & \\
\hline Trochilidae & Beija flor & Phaetornis pretrei & $\mathrm{X}$ & $\mathrm{X}$ & $\mathrm{X}$ & & $\mathrm{X}$ \\
\hline Trochilidae & B. F. tesoura & Eupetonema macroura & & $\mathrm{X}$ & & & \\
\hline Trochilidae & B. F. preto & Florisuga fusca & $\mathrm{X}$ & & & & \\
\hline Trochilidae & B. F. violeta & Colibri serrirostris & & $\mathrm{x}$ & & & $\mathrm{X}$ \\
\hline Trochilidae & Besourinho & Chlorostilbon lucidus & $\mathrm{X}$ & $\mathrm{X}$ & $\mathrm{X}$ & $\mathrm{X}$ & $\mathrm{X}$ \\
\hline Trochilidae & Beija flor & Thalurania glaucopis & & & $\mathrm{X}$ & & \\
\hline Trochilidae & Beija flor & Amazilia fimbriata & $\mathrm{x}$ & & & & \\
\hline Trochilidae & Beija flor & Amazilia lactea & $\mathrm{X}$ & $\mathrm{X}$ & $\mathrm{X}$ & $\mathrm{X}$ & $\mathrm{X}$ \\
\hline Trochilidae & Beija flor & Heliomaster squamosus & $\mathrm{X}$ & & & & $\mathrm{X}$ \\
\hline Trochilidae & Beija flor & Calliphlox amethystina & & & & & $\mathrm{X}$ \\
\hline Trogonidae & Surucuá & Trogon surrucura & $\mathrm{x}$ & & & & \\
\hline Alcedinidae & Martin pescador & Megaceryle torquata & & & $\mathrm{X}$ & & \\
\hline Alcedinidae & Martim pescador & Chloroceryle americana & & & $\mathrm{x}$ & & \\
\hline Momotidae & Juruva verde & Baryphthengus ruficapillus & $\mathrm{X}$ & & & & \\
\hline Galbulidae & Aribamba & Galbula ruficauda & $\mathrm{X}$ & & $\mathrm{X}$ & & \\
\hline Bucconidae & João bobo & Nystalus chacuru & & $\mathrm{X}$ & & $\mathrm{x}$ & \\
\hline Bucconidae & João barbudo & Malacoptila atriata & $\mathrm{x}$ & & & & \\
\hline Ramphastidae & Tucano & Ramphasto toco & $\mathrm{x}$ & $\mathrm{X}$ & $\mathrm{x}$ & $\mathrm{x}$ & $\mathrm{X}$ \\
\hline Picidae & Pica pauzinho & Picumnus cirratus & $\mathrm{X}$ & & $\mathrm{x}$ & & $\mathrm{X}$ \\
\hline Picidae & Pica pau branco & Melanerpes candidus & & $\mathrm{x}$ & & $\mathrm{x}$ & \\
\hline Picidae & Pica pau & Veniliornis passerinus & & $\mathrm{X}$ & & & \\
\hline Picidae & Pica pau & Colaptes melanochloros & $\mathrm{X}$ & $\mathrm{X}$ & $\mathrm{X}$ & & \\
\hline Picidae & Pica pau & Colaptes campestris & & $\mathrm{x}$ & $\mathrm{x}$ & $\mathrm{x}$ & $\mathrm{X}$ \\
\hline Picidae & Pica pau & Celeus flavescens & $\mathrm{x}$ & & & & \\
\hline Picidae & Pica pau & Dryocopus lineatus & $\mathrm{X}$ & & & & \\
\hline Thamnophilidae & Borralha & Mackenziana leachii & $\mathrm{x}$ & & & & \\
\hline Thamnophilidae & Choca barrada & Thamnophilus doliatus & $\mathrm{X}$ & & & & \\
\hline Thamnophilidae & Choca da mata & Thamnophilus caerulescens & $\mathrm{X}$ & & $\mathrm{X}$ & $\mathrm{X}$ & \\
\hline Thamnophilidae & Papa toaca & Pyriglena leucoptera & $\mathrm{x}$ & & $\mathrm{X}$ & & $\mathrm{X}$ \\
\hline Thamnophilidae & Choquinha lisa & Dysithamnus mentalis & $\mathrm{x}$ & & & & \\
\hline Conopophagidae & Chupa dente & Conopophaga lineata & $\mathrm{x}$ & & & & \\
\hline Dendrocolaptidae & Arapaçu & Sittasomus griseicapillus & $\mathrm{x}$ & & & & \\
\hline Dendrocolaptidae & Arapaçu & Dendrocolaptes platyrostris & $\mathrm{X}$ & & & & \\
\hline Dendrocolaptidae & Arapaçu & Leptocolaptes angustirostris & $\mathrm{X}$ & $\mathrm{X}$ & $\mathrm{X}$ & $\mathrm{X}$ & $\mathrm{X}$ \\
\hline Furnaridae & Joao de barro peq. & Furnarius figulus & & $\mathrm{X}$ & & & \\
\hline Furnaridae & Joao de barro & Furnarius rufus & & $\mathrm{x}$ & $\mathrm{X}$ & $\mathrm{x}$ & \\
\hline Furnaridae & Tifli & Synallaxis frontalis & $\mathrm{X}$ & $\mathrm{x}$ & $\mathrm{X}$ & & $\mathrm{X}$ \\
\hline Furnaridae & Ui-pi & Synallaxis albescens & $\mathrm{X}$ & & & & \\
\hline Furnaridae & João tenenem & Synallaxis spixis & & $\mathrm{X}$ & & $\mathrm{X}$ & $\mathrm{X}$ \\
\hline Furnaridae & Arredio & Cranioleuca pallida & $\mathrm{X}$ & & & & \\
\hline
\end{tabular}

Continua... 
Revista Agrogeoambiental - Dezembro/2010

Tabela 1, cont.

\begin{tabular}{|c|c|c|c|c|c|c|c|}
\hline Família & Nome popular & Nome científico & Matas Semideciduais & Áreas abertas & Mata ciliar & cerrado sensu stricto & Afloramento rochoso \\
\hline Furnaridae & Joao graveto & Phacellodomus ruffirons & & $\mathrm{x}$ & $\mathrm{x}$ & & \\
\hline Furnaridae & Cochicho & Anumbius aпnumbi & & & & & $\mathrm{x}$ \\
\hline Furnaridae & Limpa folha & Philydor rufum & $\mathrm{x}$ & & $\mathrm{x}$ & & \\
\hline Furnaridae & Barranqueiro & Automolus leucophthalmus & & & $\mathrm{x}$ & & \\
\hline Furnaridae & Bico virado & Xenops rutilans & $\mathrm{x}$ & & & & \\
\hline Furnaridae & Joao porca & Lochmias nematura & & & $\mathrm{x}$ & & \\
\hline Tyrannidae & Abre asa & Mionectes rufiventris & $\mathrm{x}$ & & & & \\
\hline Tyrannidae & Cabeçudo & Leptopogon amaurocephalus & $\mathrm{x}$ & & $\mathrm{x}$ & & $\mathrm{x}$ \\
\hline Tyrannidae & Tachuri & Hemitriccus nidipendulus & $\mathrm{x}$ & & & & \\
\hline Tyrannidae & Teque teque & Todiroatrum poliocephalum & $\mathrm{x}$ & $\mathrm{x}$ & $\mathrm{x}$ & & $\mathrm{x}$ \\
\hline Tyrannidae & Guaracava & Elaenia flavogasrer & $\mathrm{x}$ & $\mathrm{x}$ & $\mathrm{x}$ & $\mathrm{x}$ & $\mathrm{x}$ \\
\hline Tyrannidae & Guaracava & Elaenia obscura & & & $\mathrm{x}$ & & \\
\hline Tyrannidae & Risadinha & Camptostoma obsoletum & $\mathrm{x}$ & $\mathrm{x}$ & $\mathrm{x}$ & $\mathrm{x}$ & $\mathrm{x}$ \\
\hline Tyrannidae & Joao pobre & Serpophaga nigricans & & & $\mathrm{x}$ & & \\
\hline Tyrannidae & Alegrinho & Seporphaga subcristata & & $\mathrm{x}$ & $\mathrm{x}$ & & $\mathrm{x}$ \\
\hline Tyrannidae & Bagageiro & Phaeomyas murina & $\mathrm{x}$ & & & & \\
\hline Tyrannidae & Borboletinha & Phyloscartes ventralis & $\mathrm{x}$ & & & & \\
\hline Tyrannidae & Bico chato & Tolmomyas sulphurescens & $\mathrm{x}$ & & $\mathrm{x}$ & & \\
\hline Tyrannidae & Patinho & Platyrinchus mystaceus & $\mathrm{x}$ & & & & \\
\hline Tyrannidae & Felipe & Myophobus fasciatus & $\mathrm{x}$ & & & & \\
\hline Tyrannidae & Gibão & Hirundinea ferruginea & & $\mathrm{x}$ & & $\mathrm{x}$ & $\mathrm{x}$ \\
\hline Tyrannidae & Enferrujado & Lathrotriccus euleri & & & $\mathrm{x}$ & & \\
\hline Tyrannidae & Príncipe & Pyrocephalus rubinus & & & $\mathrm{x}$ & & \\
\hline Tyrannidae & Maria preta & Knipolegus lophotes & & $\mathrm{x}$ & & $\mathrm{x}$ & $\mathrm{x}$ \\
\hline Tyrannidae & Suiriri pequeno & Satrapa icterophrys & & $\mathrm{x}$ & & & \\
\hline Tyrannidae & Primavera & Xolmis cinereus & & $\mathrm{x}$ & & & $\mathrm{x}$ \\
\hline Tyrannidae & Novinha branca & Xolmis velatus & & $\mathrm{x}$ & & & \\
\hline Tyrannidae & Tesoura do brejo & Gubernetes yetapa & & $\mathrm{x}$ & $\mathrm{x}$ & & \\
\hline Tyrannidae & Lavadeira & Fluvicola nengeta & & $\mathrm{x}$ & $\mathrm{x}$ & & \\
\hline Tyrannidae & Freirinha & Arundinicola leucocephala & & & $\mathrm{x}$ & & \\
\hline Tyrannidae & Viuvinha & Colônia colonus & & $\mathrm{x}$ & $\mathrm{x}$ & & $\mathrm{x}$ \\
\hline Tyrannidae & Suiriri cavaleiro & Machetornis rixosa & & $\mathrm{x}$ & & & \\
\hline Tyrannidae & Bentevizinho & Myozetetes similis & $\mathrm{x}$ & $\mathrm{x}$ & $\mathrm{x}$ & & \\
\hline Tyrannidae & Bem te ví & Pitangus sulphuratus & & $\mathrm{x}$ & & $\mathrm{x}$ & $\mathrm{x}$ \\
\hline Tyrannidae & Bem te ví & Myodynastes maculatus & $\mathrm{x}$ & $\mathrm{x}$ & $\mathrm{x}$ & & \\
\hline Tyrannidae & Bem te vi & Megarynchus pitangua & & $\mathrm{x}$ & $\mathrm{x}$ & & \\
\hline Tyrannidae & Peitica & Empidonomus varius & $\mathrm{x}$ & & & & \\
\hline Tyrannidae & Peitica & Griseotyrannus aurantiocristatus & & $\mathrm{x}$ & & & \\
\hline Tyrannidae & Suiriri & Tyrannus albugularis & & $\mathrm{x}$ & $\mathrm{x}$ & & \\
\hline Tyrannidae & Suiriri & Tyranmus melancholicus & $\mathrm{x}$ & $\mathrm{x}$ & $\mathrm{x}$ & $\mathrm{x}$ & $\mathrm{x}$ \\
\hline Tyrannidae & Tesourinha & Tyrannus savana & & $\mathrm{x}$ & $\mathrm{x}$ & $\mathrm{x}$ & $\mathrm{x}$ \\
\hline Tyrannidae & Irré & Myarchus swainsoni & & & $\mathrm{x}$ & & \\
\hline Tyrannidae & $\mathrm{M}^{\mathrm{a}}$ cavaleira & Myarchus ferox & $\mathrm{x}$ & $\mathrm{x}$ & $\mathrm{x}$ & $\mathrm{x}$ & $\mathrm{x}$ \\
\hline Tyrannidae & $\mathrm{M}^{\mathrm{a}}$ cavaleira & Myarchus tyrannulus & & & $\mathrm{x}$ & & \\
\hline Pipridae & Tangarazinho & Ilicura militaris & $\mathrm{x}$ & & & & \\
\hline Pipridae & Tangará & Chiroxiphia caudata & $\mathrm{x}$ & & $\mathrm{x}$ & & \\
\hline Tityridae & Flautim & Schiffornis virescens & $\mathrm{x}$ & & & & \\
\hline Tityridae & Anambé & Tytira inquisitor & & & & & $\mathrm{x}$ \\
\hline Tityridae & Caneleiro & Pachyramphus polychopterus & $\mathrm{x}$ & & $\mathrm{x}$ & & \\
\hline Vieronidae & Pitiguari & Cyclarhis guajanensis & $\mathrm{x}$ & & $\mathrm{x}$ & & \\
\hline Vieronidae & Juruviara & Vireo olivaceus & $\mathrm{x}$ & & & & \\
\hline Vieronidae & Vite vite & Hylophilus amaurocephalus & $\mathrm{x}$ & & & & \\
\hline Corvidae & Gralha do cerrado & Cyanocorax cristatelus & & & $\mathrm{x}$ & $\mathrm{x}$ & $\mathrm{x}$ \\
\hline Corvidae & Gralha chanchã & Cyanocorax crisops & & $\mathrm{x}$ & & & \\
\hline Hirundinidae & Andorinha & Tachycineta leucorrhoa & & $\mathrm{x}$ & & & \\
\hline Hirundinidae & Andorinha & Progne chalubea & & $\mathrm{x}$ & & & \\
\hline
\end{tabular}

Continua... 
Lista preliminar da avifauna da A.P.A. Coqueiral e primeiro

registro de Tytira inquisitor no Sul de Minas Gerais, Brasil

Tabela 1, cont.

\begin{tabular}{|c|c|c|c|c|c|c|c|}
\hline Família & Nome popular & Nome científico & $\begin{array}{c}\text { Matas } \\
\text { Semideciduais }\end{array}$ & $\begin{array}{l}\begin{array}{l}\text { Áreas } \\
\text { abertas }\end{array} \\
\end{array}$ & Mata ciliar & $\begin{array}{l}\text { cerrado } \\
\text { sensu stricto }\end{array}$ & $\begin{array}{l}\text { Afloramento } \\
\text { rochoso }\end{array}$ \\
\hline Hirundinidae & Andorinha & Pygochelidon cyanoleuca & & $\mathrm{X}$ & & & \\
\hline Hirundinidae & Andorinha & Stelgidopteryx ruficollis & & $\mathrm{X}$ & $\mathrm{x}$ & & $\mathrm{X}$ \\
\hline Hirundinidae & Andorinha & Riparia riparia & & & $\mathrm{x}$ & & $\mathrm{X}$ \\
\hline Troglodytidae & Curruira & Troglodytes musculus & & $\mathrm{X}$ & & & $\mathrm{X}$ \\
\hline Donacobiidae & Japacanim & Donacobius atricapilla & & & $\mathrm{x}$ & & \\
\hline Turdidae & Sabiá laranjeira & Turdus rufiventris & $\mathrm{X}$ & $\mathrm{X}$ & $\mathrm{x}$ & & $\mathrm{X}$ \\
\hline Turdidae & Sabiá barranco & Turdus leucomelas & $\mathrm{X}$ & $\mathrm{X}$ & $\mathrm{x}$ & $\mathrm{X}$ & $\mathrm{x}$ \\
\hline Turdidae & Sabiá poça & Turdus amaurochalinus & $\mathrm{X}$ & $\mathrm{x}$ & $\mathrm{x}$ & & $\mathrm{X}$ \\
\hline Turdidae & Sabiá coleira & Turdus albicollis & $\mathrm{X}$ & & & & \\
\hline Mimidae & Sabiá do campo & Mimus saturninus & & $\mathrm{X}$ & & & $\mathrm{X}$ \\
\hline Motacillidae & Caminheiro & Anthus lutescens & & & & & $\mathrm{X}$ \\
\hline Coerebidae & Cambacica & Coereba flaveola & $\mathrm{X}$ & $\mathrm{x}$ & $\mathrm{x}$ & $\mathrm{x}$ & $\mathrm{x}$ \\
\hline Thraupidae & Bico de veludo & Schitochlamys ruficapillus & & & & & $\mathrm{X}$ \\
\hline Thraupidae & Tietinga & Cissopis leverianus & $\mathrm{X}$ & & & & \\
\hline Thraupidae & Saira & Nemosia pileata & $\mathrm{X}$ & $\mathrm{X}$ & $\mathrm{X}$ & & \\
\hline Thraupidae & Saira canario & Thlypopsis sordida & $\mathrm{X}$ & & & & \\
\hline Thraupidae & Tié preto & Tachyphonus caronatus & $\mathrm{X}$ & $\mathrm{x}$ & $\mathrm{x}$ & $\mathrm{X}$ & $\mathrm{X}$ \\
\hline Thraupidae & Sanhaço & Thraupis sayaca & $\mathrm{X}$ & $\mathrm{x}$ & $\mathrm{x}$ & $\mathrm{X}$ & $\mathrm{X}$ \\
\hline Thraupidae & Sanhaço & Thraupis palmarum & $\mathrm{X}$ & & & & \\
\hline Thraupidae & Anhaço bandeira & Tangara cayana & $\mathrm{x}$ & $\mathrm{x}$ & $\mathrm{x}$ & $\mathrm{x}$ & $\mathrm{x}$ \\
\hline Thraupidae & Saira dorada & Tangara cyanoventris & $\mathrm{X}$ & & & & \\
\hline Thraupidae & Saí andorinha & Tersina viridis & $\mathrm{x}$ & $\mathrm{x}$ & $\mathrm{x}$ & & $\mathrm{X}$ \\
\hline Thraupidae & Saí azul & Dacnis cayana & $\mathrm{X}$ & $\mathrm{x}$ & $\mathrm{x}$ & & \\
\hline Thraupidae & Saíra ferrugem & Hemithraupis ruficapilla & $\mathrm{X}$ & & & & \\
\hline Thraupidae & Figuinha & Conirostrum speciosum & $\mathrm{X}$ & & $\mathrm{x}$ & & \\
\hline Emberizidae & Tico tico & Zonotrichia capensis & & $\mathrm{x}$ & $\mathrm{x}$ & $\mathrm{X}$ & $\mathrm{X}$ \\
\hline Emberizdae & Tico tico do campo & Ammodramus humerallis & & $\mathrm{x}$ & & & $\mathrm{X}$ \\
\hline Emberizdae & Canário da terra & Sicalis flaveola & & $\mathrm{x}$ & & & \\
\hline Emberizidae & Sabiá do banhado & Embernagra platensis & & $\mathrm{x}$ & $\mathrm{x}$ & & \\
\hline Emberizidae & Tiziu & Volatinia jacarina & & $\mathrm{x}$ & & $\mathrm{X}$ & $\mathrm{X}$ \\
\hline Emberizidae & Bigodinho & Sporophila lineola & & $\mathrm{x}$ & $\mathrm{x}$ & & \\
\hline Emberizdae & Cabeça preta & Sporophila nigricollis & & $\mathrm{x}$ & & $\mathrm{X}$ & $\mathrm{X}$ \\
\hline Emberizidae & Coleirinha & Sporophila caerulescen & & $\mathrm{x}$ & & $\mathrm{x}$ & $\mathrm{X}$ \\
\hline Emberizidae & Patativo & Sporophila leucoptera & & & $\mathrm{x}$ & & \\
\hline Emberizidae & Tico tico da mata & Arremom flavirostris & $\mathrm{X}$ & & & & \\
\hline Emberizidae & Soldadinho & Coryphospingus pilleatus & & $\mathrm{x}$ & $\mathrm{x}$ & $\mathrm{X}$ & $\mathrm{x}$ \\
\hline Cardinalidae & Trinca ferro & Saltator similis & $\mathrm{X}$ & $\mathrm{x}$ & $\mathrm{x}$ & $\mathrm{x}$ & $\mathrm{X}$ \\
\hline Cardinalidae & Azulão & Cyanocompsa brissonii & & & $\mathrm{x}$ & & \\
\hline Parulidae & Mariquita & Parula pitiayumi & $\mathrm{X}$ & & & & $\mathrm{X}$ \\
\hline Parulidae & Pia cobra & Geothypis aequinoctialis & $\mathrm{X}$ & & & & \\
\hline Parulidae & Pula pula & Basileuterus culicivorus & $\mathrm{X}$ & & & & \\
\hline Parulidae & Pula pula & Basileuterus hypoleucus & $\mathrm{X}$ & & $\mathrm{x}$ & & \\
\hline Parulidae & Pula pula & Basileuterus flaveolus & $\mathrm{X}$ & & & & $\mathrm{X}$ \\
\hline Icteridae & Japú & Psarocolius decumanus & $\mathrm{X}$ & $\mathrm{X}$ & $\mathrm{x}$ & & $\mathrm{X}$ \\
\hline Icteridae & Pássaro preto & Gnorimopsar chopi & & $\mathrm{X}$ & & & $\mathrm{X}$ \\
\hline Icteridae & Garibaldi & Chrysomus ruficapillus & & & $\mathrm{x}$ & & \\
\hline Icteridae & Chopim do brejo & Pseudoleiste guirauro & & $\mathrm{X}$ & & & \\
\hline Icteridae & Chopim & Molothrus bonariensis & & $\mathrm{x}$ & & & $\mathrm{X}$ \\
\hline Frigillidae & Pintassilgo & Carduelis magellanica & & $\mathrm{X}$ & & & $\mathrm{X}$ \\
\hline Frigillidae & Fi-fi & Euphonia chlorotica & $\mathrm{X}$ & $\mathrm{X}$ & $\mathrm{X}$ & & $\mathrm{X}$ \\
\hline Passeridae & Pardal & Passer domesticus & & $\mathrm{X}$ & & & \\
\hline TOTAL & & 205 espécies & & & & & \\
\hline
\end{tabular}

